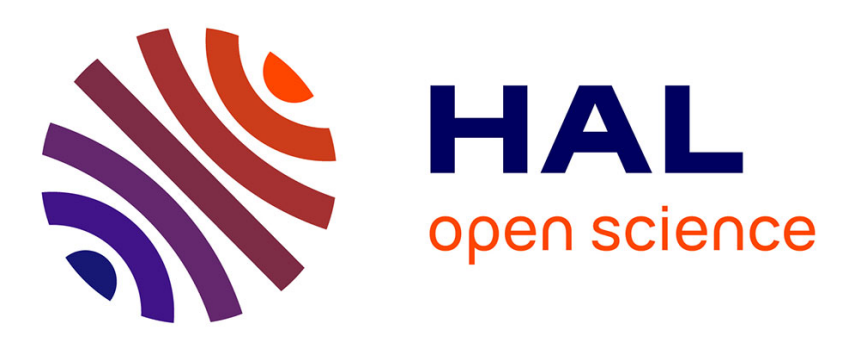

\title{
An implicit numerical method for wear modeling applied to a hip joint prosthesis problem \\ Franck Jourdan, Amine Samida
}

\section{To cite this version:}

Franck Jourdan, Amine Samida. An implicit numerical method for wear modeling applied to a hip joint prosthesis problem. Computer Methods in Applied Mechanics and Engineering, 2009, 198 (2729), pp.2209-2217. 10.1016/j.cma.2009.02.017 . hal-00686709

\section{HAL Id: hal-00686709 \\ https://hal.science/hal-00686709}

Submitted on 11 Apr 2012

HAL is a multi-disciplinary open access archive for the deposit and dissemination of scientific research documents, whether they are published or not. The documents may come from teaching and research institutions in France or abroad, or from public or private research centers.
L'archive ouverte pluridisciplinaire HAL, est destinée au dépôt et à la diffusion de documents scientifiques de niveau recherche, publiés ou non, émanant des établissements d'enseignement et de recherche français ou étrangers, des laboratoires publics ou privés. 


\title{
An implicit numerical method for wear modeling applied to a hip joint prosthesis problem
}

\author{
Franck Jourdan, Amine Samida
}

December 20, 2008

Laboratoire de Mécanique et Génie Civil, Université Montpellier 2/CNRS UMR 5508, Place

Eugène Bataillon, CC 048, F-34095 Montpellier Cedex 5, France

\begin{abstract}
Some phenomenological wear models exist, but they are most of the time used to post-process numerical simulations. In certain situations however, material loss may be sufficient to change the contact area, contact stresses. In such cases, coupling the wear loss estimation to the mechanical contact modelling becomes essential. It can be done, and is done most of the time, by updating geometry between timesteps, introducing no further non-linearity. The option chosen here is on the contrary a fully coupled model where the wear displacement field is added to the unknowns of the frictional contact problem and requires one more equation. This equation is an adaptation of the $\mathrm{Ar}-$ chard's wear law to a local formulation in the context of dynamic and
\end{abstract}


large displacements applications. This method allows simulation of the entire wear process in only a few loading cycles. We even quite accurately simulate the process in a single cycle. This is made possible via our fully coupled approach and an adaptation of the wear factor. The wear factor is artificially increased in order to decrease the number of cycles. A detailed description of the resolution of discrete equations is presented. The solver is a non linear Gauss Seidel algorithm adapted to wear conditions which derives from the 'Non Smooth Contact Dynamics' method. The wear model is validated on a total hip arthroplasty problem. Numerical observations are based on previously published experimental results.

Keywords : 3D, wear model, contact, friction, dynamic, implicit, hip, prostheses

\section{Introduction}

Wear is a problem that arises in many industrial applications, but also in everyday life. Solutions are required in many areas to reduce wear on mechanisms. The tribology of bodies in contact must be optimized by improving the coating surfaces, which thus concerns Material Science. It is also essential to analyze and optimize the geometry of parts in contact. The present study is in this field of expertise. We propose a numerical tool to assist in the design of products to reduce the effects of wear. There are many articles on wear modeling in the literature. Two models type have been described. 
In the first model type, the set of resulting wear fragments is considered as a third body [10], [31], [6] while the second model type does not take these wear fragments into account [3], [28], [20]. The influence of the third body is taken into account through the introduction of a macroscopic wear factor. One of the main reasons of these choice is to reduce the computational time. In most articles, [11], [20], [22], [18], [27], to save more computational time, wear is computed after several time steps and the geometry is updated. Our method is an alternative to the preceding ones. Contrary to them, we propose a fully coupled model in which wear and geometry are computed at each iteration of each time step. The aim of this method is to simulate the wear process in just a few cycles, by using an artificial amplification of the wear factor.

This article is a follow-up of the paper [15]. We describe a generalization to three-dimensional problems of wear in dynamic and large displacements context. The third body is not directly considered in this approach. Wear behavior is controlled by an extension of Archard's law [5]. Matter loss, due to wear, is related to the normal pressure and sliding velocity. It is thus essential to accurately reproduce the kinematics of the bodies in contact, but also the applied forces.

The method deals with unilateral signorini's conditions and the dry friction law of Coulomb. A numerical treatment is developed in this paper. The discretized frictional contact problem is solved using a non linear Gauss Sei- 
del algorithm and derives from the 'Non Smooth Contact Dynamics' method, developed by J.J Moreau and M. Jean [13]. The non linearities come from the frictional contact operator which deals with the wear conditions. Here we focus on the method developed to solve the three-dimensional problem.

Finally, we validate our numerical method on a problem of hip prosthesis wear.

\section{Wear modeling}

The wear model is discussed and further described in [15]. Here we just review the main relationships. The wear model is built to deal with large strain and dynamic effects. It is a macroscopic model. The microscopic effects like asperities deformations and material tearing are not directly considered. The influence of these microscopic phenomena are taken into account through a macroscopic wear factor. The temperature effects are neglected, based on the assumption that the temperature in invivo joint prostheses, are sufficiently small.

Let $\Omega_{O}$ be the domain at time $t=0$ and $\underline{\mathrm{X}}$ be the initial position of a particle of the body, then the transformation operator $\underline{\varphi}$ gives the position $\underline{\mathrm{x}}$ of the particle at time $t$, as follows

$$
\underline{x}=\underline{\varphi}(\underline{X}, t)
$$


Figure 1: Local frame

The transformation operator $\underline{\varphi}$ is split in two parts.

$$
\underline{\varphi}(\underline{X}, t)=\underline{\varphi}^{e}(\underline{X}, t)+\underline{\varphi}^{w}(\underline{X}, t)
$$

where $\underline{\varphi}^{w}$ is the transformation due to wear and $\underline{\varphi}^{e}$ is the complementary part. The transformation $\underline{\varphi}^{w}$ is assumed to be nil beyond the contact zone. Before developing the evolution of the transformation due to the wear in the contact zone, it is necessary to introduce some notations relative to contact conditions. We consider two candidate contact bodies. Vectors $\underline{T}_{1}$ and $\underline{T}_{2}$ of the local frame $\left(\underline{T}_{1}, \underline{T}_{2}, \underline{N}\right)$ are chosen in the common tangent plane of the two bodies. Vector $\underline{N}$ is the normal vector at the tangent plane such that $\left(\underline{T}_{1}, \underline{T}_{2}, \underline{N}\right)$ is orthonormal (see Figure 1).

Let $\underline{\varphi}_{1}$ be the transformation operator of the first body, and $\underline{\varphi}_{2}$ the transformation operator of the second body. The tangential relative velocity $\underline{v}_{T}$ is defined as the projection of the relative velocity vector $\underline{v}$ on the common tangent plane:

$$
\underline{v}=\underline{\dot{\varphi}}_{1}-\underline{\dot{\varphi}}_{2}
$$

Let $\underline{r}=\left(r_{T 1}, r_{T 2}, r_{N}\right)$ be the reaction force exerted on the first body, expressed in the local frame, the normal component of velocity due to the wear, is assumed to be governed by the following relationship :

$$
\left\{\begin{array}{l}
\frac{d}{d t}\left(\underline{\varphi}_{1}^{w} \cdot \underline{N}\right)=k_{1}^{w} r_{N}\left|\underline{v}_{T}\right| \\
\frac{d}{d t}\left(\underline{\varphi}_{2}^{w} \cdot \underline{N}\right)=-k_{2}^{w} r_{N}\left|\underline{v}_{T}\right|
\end{array}\right.
$$


where $k_{i}^{w}$ is the local wear factor of the body $i=1,2$. The tangential component is assumed to be nil.

$$
\underline{\varphi}_{i}^{w} \cdot \underline{T}_{i}=0 \quad \forall i=1,2
$$

This wear law has to be considered in the context of frictional contact laws. Unilateral Signorini's conditions and the dry friction law of Coulomb were chosen for this study. If $g_{N}=\left(\underline{\varphi}_{1}-\underline{\varphi}_{2}\right) \cdot \underline{N}$ denotes the gap between the two bodies, the unilateral Signorini's conditions can be expressed with the following relationships

$$
\left\{\begin{array}{c}
r_{N} \geq 0 \\
g_{N} \geq 0 \\
g_{N} r_{N}=0
\end{array}\right.
$$

or in the equivalent variational equation form

$$
r_{N}=\operatorname{proj}_{\Re^{+}}\left(r_{N}-\rho_{n} g_{N}\right)
$$

where $\operatorname{proj}_{\Re^{+}}$denotes the orthogonal projection on the positive real set $\Re^{+}$ and $\rho_{N}$ is a positive definite real number.

Let $\underline{r}_{T}$ be the tangential part of the reaction force, then the Coulomb law is governed by the following relationships

$$
\left\{\begin{array}{c}
\left\|\underline{r}_{T}\right\| \leq \mu r_{N} \\
\left\|\underline{r}_{T}\right\|<\mu r_{N} \quad \Rightarrow \quad \underline{v}_{T}=0 \\
\underline{v}_{T} \neq 0 \quad \Rightarrow \quad \underline{r}_{T}=-\mu r_{N} \frac{\underline{v}_{T}}{\left\|\underline{v}_{T}\right\|}
\end{array}\right.
$$


where $\mu$ is the friction coefficient. These relationships are equivalent to the next equation

$$
\underline{r}_{T}=\operatorname{proj}_{C\left(r_{N}\right)}\left(\underline{r}_{T}-\rho_{T} \underline{v}_{T}\right)
$$

where $C\left(r_{N}\right)$ is the section of the Coulomb cone $\left\{\underline{r}_{T} \in \Re^{2}\right.$ such that $\left.\left\|\underline{r}_{T}\right\| \leq \mu r_{N}\right\}$, and $\rho_{T}$ is a positive definite real number. The parameters $\rho_{N}$ and $\rho_{T}$ are neither Lagrange multipliers, nor penalty coefficients. Their values are not necessarily large and do not have physical significances. However, for numerical reasons, they have optimal values (see end of paragraph 3.2)

\section{Numerical treatment}

In this section, we present the numerical method used to solve the motion equation of a body under contact, friction and wear conditions. This method is based on the "Non Smooth Contact Dynamics" method proposed by J.J. Moreau and M. Jean [13]. This method was initially developed for rigid bodies and applied on deformable bodies in [17], [1]. It is shown in [16], that it is a non linear Gauss Seidel algorithm.

\subsection{Non Smooth Contact Dynamics (NSCD)}

In this paragraph, we propose an adaptation of the NSCD method to wear conditions.

In order to simplify the presentation, body $S 2$ is supposed to be rigid 
and the method is presented to solve the motion equation of body $S 1$. In the context of large displacements, we use a total lagrangian approach. In this formulation, the virtual principal power can be written, in the referential configuration, as follows

$$
\begin{gathered}
\int_{\Omega_{0}} \rho_{0} \underline{\ddot{x}} \cdot \underline{v}^{*} d X+\int_{\Omega_{0}} \operatorname{tr}\left(\underline{\underline{P}}^{T} \underline{\underline{\nabla v}}^{*}\right) d X=\int_{\partial_{1} \Omega_{0}} \underline{F}_{d}^{0} \cdot \underline{v}^{*} d S+\int_{\Omega_{0}} \underline{f}_{d}^{0} \underline{.}^{*} d X \\
\forall t \in[0, T] \text { and } \forall \underline{\mathrm{v}}^{*} \in \mathrm{U}_{\mathrm{ad}}^{0}=\left\{\underline{\mathrm{v}}^{*},\left.\underline{\mathrm{v}}^{*}\right|_{\partial_{0} \Omega_{0}}=0\right\}
\end{gathered}
$$

where $\rho_{0}$ is the specific mass on the referential configuration and $\underline{\underline{P}}$ is the first tensor of Piola-Kirchhoff. The tensor $\underline{\underline{P}}$ is defined by

$$
\underline{\underline{P}}=J \underline{\underline{\sigma}} \underline{\underline{F}}^{-T}
$$

where $\underline{\underline{F}}=\underline{\underline{\nabla}} \varphi$ is the gradient of the transformation $\underline{\varphi}, J=\operatorname{det}(\underline{\underline{F}})$ and $\underline{\underline{\sigma}}$ is the Cauchy stress tensor.

The space discretization of the motion equation is obtained using the finite element method. For each finite element $I$ the position $\underline{x}$ of a particle at time $t$ is interpolated as follows:

$$
\underline{x}=N^{I}(\underline{X}) q_{I}^{-}
$$

where $N^{I}$ is the matrix of interpolation functions and $q_{I}^{-}$is the vector of node positions of element $I$ at time $t$.

Coupling a $\theta$-method for the time discretization with the previous spacial interpolation, the non linear system (8) becomes $M\left(q^{+}\right)\left(\dot{q}^{+}-\dot{q}^{-}\right)-h \theta\left(F_{\text {int }}\left(q^{+}\right)+F_{\text {ext }}^{+}\right)-h(1-\theta)\left(F_{\text {int }}\left(q^{-}\right)+F_{\text {ext }}^{-}\right)-h R^{+}=0$ 
where $h$ is the time step, $M\left(q^{+}\right)$is the mass matrix, $q^{+}$is the generalized vector of the node positions at time $t+h, \dot{q}^{+}$is the generalized vector of the node velocity at time $t+h, F_{\text {int }}\left(q^{+}\right)$is the generalized vector of internal forces, $R^{+}$is the generalized vector of reaction forces at time $t+h$, and $F_{e x t}^{+}$ is the generalized vector of given external forces at time $t+h$. Subscript "-" denotes values at time $t$ and subscript " + " denotes values at time $t+h$.

Based on the wear law (4) and splitting (2), $q^{+}$is a function of the elastic part $q^{e+}$ of nodal positions and the vector of nodal reaction forces $R^{+}$:

$$
q^{+}=q^{e+}+q^{w+}=f_{w}\left(q^{e+}, R^{+}\right)
$$

The function $f_{w}$ cannot be expressed explicitly. The internal forces are only assumed to depend on the elastic part of the deformation :

$$
F_{\text {int }}\left(q^{+}\right)=F_{\text {int }}\left(q^{e+}\right)
$$

The system of equations (11) is linearized using a modified Newton method, applied on the variable $\dot{q}^{e+}$. Then, $\dot{q}^{e+}$ is found to be the limit of sequence $\left(\dot{q}_{k}^{e+}\right)_{k \in N}$ such that

$$
\begin{aligned}
& \left(M+h^{2} \theta^{2} K\right)\left(\dot{q}_{k+1}^{e+}-\dot{q}_{k}^{e+}\right)-h R_{k+1} \\
& =-M\left(\dot{q}_{k}^{+}-\dot{q}^{-}\right)-h \theta\left[F_{\text {int }}\left(q_{k}^{e+}\right)-F_{\text {ext }}^{+}\right]-h(1-\theta)\left[F_{\text {int }}\left(q^{e-}\right)-F_{\text {ext }}^{-}\right] \\
& \quad \text { where } K=\frac{\partial F_{\text {int }}}{\partial q^{e+}} .
\end{aligned}
$$


This is a modified Newton method, because the gradient $\frac{\partial f_{w}}{\partial q^{e+}}$ is not considered in the tangent operator. Moreover, the mass variation $\frac{\partial M}{\partial q^{e+}}$ is neglected.

Using

$$
K_{T}=\left(M+h^{2} \theta^{2} K\right)
$$

the equation (14) becomes:

$$
\dot{q}_{k+1}^{e+}-\dot{q}_{k}^{e+}-h W R_{k+1}=V_{k}
$$

with:

$W=K_{T}^{-1}$

$V_{k}=K_{T}^{-1}\left[-M\left(\dot{q}_{k}^{+}-\dot{q}^{-}\right)-h \theta F_{\text {int }}\left(q_{k}^{e+}\right)-h(1-\theta) F_{\text {int }}\left(q^{e-}\right)+h \theta F_{\text {ext }}^{+}+h(1-\theta) F_{\text {ext }}^{-}\right]$

This system (15) must be coupled with the frictional contact and wear laws. The equations are expressed in the local frames to solve this problem. For each node $\alpha$ candidate to contact, $H_{\alpha}$ denotes the change of frame matrix 
such that

$$
H_{\alpha}=\left(\begin{array}{ccccccc}
I_{3} & 0 & 0 & 0 & 0 & 0 & 0 \\
0 & \cdot & 0 & 0 & 0 & 0 & 0 \\
0 & 0 & I_{3} & 0 & 0 & 0 & 0 \\
0 & 0 & 0 & Q(\alpha) & 0 & 0 & 0 \\
0 & 0 & 0 & 0 & I_{3} & 0 & 0 \\
0 & 0 & 0 & 0 & 0 & . & 0 \\
0 & 0 & 0 & 0 & 0 & 0 & I_{3}
\end{array}\right)
$$

where $I_{3}$ is the identity matrix of $\Re^{3}$ and $Q(\alpha)$ is the $3 \times 3$ matrix of rotation between the global and local frame associated with node $\alpha$.

If $\underline{v}^{e \alpha}$ is the elastic part of the relative velocity at iteration $k+1$, then

$$
v^{e \alpha}=H_{\alpha}\left(\dot{q}_{k+1}^{e+}-\dot{q}_{0}^{e+}\right)
$$

where $\dot{q}_{0}^{e+}$ is the velocity of the antagonist points (center of the local frames). If $\underline{r}^{\alpha}$ represents the reaction forces exerted on node $\alpha$, at iteration $k+1$, we define

$$
R_{k+1}^{\alpha}=H_{\alpha}^{T} \underline{r}^{\alpha}
$$

With these new variables, we can transform equation (15) as follows

$$
\begin{aligned}
(15) & \Leftrightarrow \quad \dot{q}_{k+1}^{e+}-h W R_{k+1}^{\alpha}=h W\left(R_{k+1}-R_{k+1}^{\alpha}\right)+\dot{q}_{k}^{e+}+V_{k} \\
& \Leftrightarrow \quad H_{\alpha}\left(\dot{q}_{k+1}^{e+}-h W R_{k+1}^{\alpha}\right)=H_{\alpha}\left(h W\left(R_{k+1}-R_{k+1}^{\alpha}\right)+\dot{q}_{k}^{e+}+V_{k}\right)
\end{aligned}
$$


$\Leftrightarrow \quad H_{\alpha}\left(\dot{q}_{k+1}^{e+}-\dot{q}_{0}^{e+}-h W R_{k+1}^{\alpha}\right)=H_{\alpha}\left(h W\left(R_{k+1}-R_{k+1}^{\alpha}\right)+\dot{q}_{k}^{e+}+V_{k}-\dot{q}_{0}^{e+}\right)$

Then, for each node $\alpha$, the linearized motion equation is reduced to

$$
\underline{v}^{e \alpha}-h W^{\alpha} \underline{r}^{\alpha}=\underline{v}_{f}^{e \alpha}
$$

where $\underline{v}_{f}^{e \alpha}$ is the free elastic velocity. This is the elastic velocity that node $\alpha$ would have when the reaction force $\underline{r}^{\alpha}$ is nil and when the reaction forces of the other nodes have the previously computed values. Matrix $W^{\alpha}$ is defined by

$$
W^{\alpha}=H_{\alpha}\left(M+h^{2} \theta^{2} K\right)^{-1} H_{\alpha}^{T}
$$

On the other hand, the contact and friction relationships are

$$
r_{N}^{\alpha}=\operatorname{proj}_{\Re^{+}}\left(r_{N}^{\alpha}-\rho_{n} g_{N}^{\alpha}\right)
$$

and

$$
\underline{r}_{T}^{\alpha}=\operatorname{proj}_{C\left(r_{N}^{\alpha}\right)}\left(\underline{r}_{T}^{\alpha}-\rho_{T} \underline{v}_{T}^{e \alpha}\right)
$$

Gap $g_{N}^{\alpha}$ is governed by the following relationship

$$
g_{N}^{\alpha}=g_{N}^{\alpha-}+h(1-\theta) v_{N}^{\alpha-}+h \theta\left(v_{N}^{e \alpha}+k^{w} r_{N}^{\alpha}\left|\underline{v}_{T}^{e \alpha}\right|\right)
$$

where $g_{N}^{\alpha-}$ is the gap at time $t, v_{N}^{\alpha-}$ is the normal component of the relative velocity at time $t$ and $k^{w}=k_{1}^{w}+k_{2}^{w}$ is the local wear factor. The system of equations (18), (20), (21) is solved using a generalized Newton method. The method is described in detail in the following paragraph. Then, after 
computation of the local variables $\underline{v}_{T}^{e \alpha}, v_{N}^{e \alpha}, \underline{r}_{T}^{\alpha}, r_{N}^{\alpha}$, the next candidate contact node $\alpha+1$ is treated, and so on, until a convergence criterion is satisfied. An overview of the algorithm is given in the scheme ??

\subsection{Local solver}

The system of equations (18), (20), (21) is non linear and non differentiable. We decided to use a generalized Newton method [4]. This method is applied at the local variable $y=\left(v_{T_{1}}^{e \alpha}, v_{T_{2}}^{e \alpha}, v_{N}^{e \alpha}, r_{T_{1}}^{\alpha}, r_{T_{2}}^{\alpha}, r_{N}^{\alpha}\right)^{T} \in \Re^{6}$.

Note that operator $\operatorname{proj}_{C\left(r_{N}^{\alpha}\right)}($.$) is not defined for r_{N}^{\alpha}<0$, so it is replaced by $\operatorname{proj}_{C\left(\lambda_{N}^{+}\right)}($.$) with :$

$$
\lambda_{N}^{+}=\operatorname{proj}_{\Re^{+}}(\underbrace{r_{N}^{\alpha}-\rho_{N} g_{N}^{\alpha}}_{\lambda_{N}})
$$

The solutions of (20) is the same as of

$$
r_{N}^{\alpha}=\operatorname{proj}_{C\left(\lambda_{N}^{+}\right)}\left(\lambda_{N}\right)
$$

The variable

$$
\underline{\lambda}_{T}=\underline{r}_{T}^{\alpha}-\rho_{T} \underline{v}_{T}^{e \alpha}
$$

is also introduced, and the system of equations (18), (20), (21) can be rewritten as

$$
\mathcal{F}(y)=\mathcal{A} y+g(y)-v^{l}=0
$$


with:

$$
\mathcal{A}=\left(\begin{array}{cc}
I_{3} & -h W^{\alpha} \\
0 & -I_{3}
\end{array}\right), \quad v^{l}=\left(\begin{array}{c}
\left(v_{f}^{e \alpha}\right)_{T_{1}} \\
\left(v_{f}^{e \alpha}\right)_{T_{2}} \\
\left(v_{f}^{e \alpha}\right)_{N} \\
0 \\
0 \\
0
\end{array}\right) \text { and } \mathrm{g}(\mathrm{y})=\left\{\begin{array}{c}
0 \\
0 \\
\operatorname{proj}_{C\left(\lambda_{N}^{+}\right)}\left(\lambda_{T_{1}}\right) \\
\operatorname{proj}_{C\left(\lambda_{N}^{+}\right)}\left(\lambda_{T_{2}}\right) \\
\operatorname{proj}_{\Re^{+}}\left(\lambda_{N}\right)
\end{array}\right.
$$

The unknown $y$ is found as the limit of sequence $\left\{y_{n}\right\}_{n \in N}$ such that:

$$
\begin{aligned}
{\left[\mathcal{A}+\nabla g\left(y_{n}\right)\right]\left(y_{n+1}-y_{n}\right) } & =-\mathcal{F}\left(y_{n}\right) \\
\Leftrightarrow \quad \underbrace{\left[\mathcal{A}+\nabla g\left(y_{n}\right)\right]}_{\mathcal{F}^{\prime}} y_{n+1} & =\nabla g\left(y_{n}\right) y_{n}-g\left(y_{n}\right)+v^{l}
\end{aligned}
$$

To compute $\mathcal{F}^{\prime}$ we must distinguish to cases

1. If $\lambda_{N} \leq 0$ (no contact)

In this case $\lambda_{N}^{+}=0$ et $g(y)=(0)$

$$
\begin{aligned}
& \Rightarrow \nabla g=[0] \\
& \Rightarrow \mathcal{F}^{\prime}=\mathcal{A}
\end{aligned}
$$

2. If $\lambda_{N}>0$ : (contact)

There are two possibilities:

(a) If $\left\|\underline{\lambda}_{T}\right\| \leq \mu \lambda_{N}$ (sticking) then : 


$$
g(y)=\left\{\begin{array}{c}
0 \\
0 \\
0 \\
\lambda_{T_{1}} \\
\lambda_{T_{2}} \\
\lambda_{N}
\end{array}\right.
$$

$\Rightarrow \mathcal{F}^{\prime}=\left(\begin{array}{cccccc}1 & 0 & 0 & -h W_{11}^{\alpha} & -h W_{12}^{\alpha} & -h W_{13}^{\alpha} \\ 0 & 1 & 0 & -h W_{21}^{\alpha} & -h W_{22}^{\alpha} & -h W_{23}^{\alpha} \\ 0 & 0 & 1 & -h W_{31}^{\alpha} & -h W_{32}^{\alpha} & -h W_{33}^{\alpha} \\ -\rho_{T} & 0 & 0 & 0 & 0 & 0 \\ 0 & -\rho_{T} & 0 & 0 & 0 & 0 \\ \mathcal{F}_{61}^{\prime} & \mathcal{F}_{62}^{\prime} & -\rho_{N} h \theta & 0 & 0 & -\rho_{N} h \theta\left\|_{T}\right\|\end{array}\right)$

with

$\mathcal{F}_{61}^{\prime}=-\rho_{N} h \theta k^{w} r_{N} \frac{v_{T_{1}}}{\left\|\underline{v}_{T}\right\|}$

$\mathcal{F}_{62}^{\prime}=-\rho_{N} h \theta k^{w} r_{N} \frac{v_{T_{2}}}{\left\|\underline{v}_{T}\right\|}$

In this case the two bodies have a sticking contact status. 
(b) If $\left\|\underline{\lambda}_{T}\right\| \geq \mu \lambda_{N}$ (sliding)

then :

$$
g(y)=\left\{\begin{array}{c}
0 \\
0 \\
0 \\
\mu \lambda_{N} \frac{\lambda_{T_{1}}}{\left\|\underline{\lambda}_{T}\right\|} \\
\mu \lambda_{N} \frac{\lambda_{T_{2}}}{\left\|\underline{\lambda}_{T}\right\|} \\
\lambda_{N}
\end{array}\right.
$$

$\Rightarrow \mathcal{F}^{\prime}=\left(\begin{array}{cccccc}1 & 0 & 0 & -h W_{11}^{\alpha} & -h W_{12}^{\alpha} & -h W_{13}^{\alpha} \\ 0 & 1 & 0 & -h W_{21}^{\alpha} & -h W_{22}^{\alpha} & -h W_{23}^{\alpha} \\ 0 & 0 & 1 & -h W_{31}^{\alpha} & -h W_{32}^{\alpha} & -h W_{33}^{\alpha} \\ \mathcal{F}_{41}^{\prime} & \mathcal{F}_{42}^{\prime} & \mathcal{F}_{43}^{\prime} & \mathcal{F}_{44}^{\prime} & \mathcal{F}_{45}^{\prime} & \mathcal{F}_{46}^{\prime} \\ \mathcal{F}_{51}^{\prime} & \mathcal{F}_{52}^{\prime} & \mathcal{F}_{53}^{\prime} & \mathcal{F}_{54}^{\prime} & \mathcal{F}_{55}^{\prime} & \mathcal{F}_{56}^{\prime} \\ \mathcal{F}_{61}^{\prime} & \mathcal{F}_{62}^{\prime} & -\rho_{N} h \theta & 0 & 0 & -\rho_{N} h \theta\left\|\underline{v}_{T}\right\|\end{array}\right)$

with:

$$
\begin{aligned}
& \mathcal{F}_{41}^{\prime}=-\frac{\mu}{\left\|\underline{\lambda}_{T}\right\|}\left(\rho_{N} h \theta k^{w} r_{N} \lambda_{T_{1}} \frac{v_{T_{1}}}{\left\|\underline{v}_{T}\right\|}+\rho_{T} \lambda_{N} \frac{\lambda_{T_{2}}^{2}}{\left\|\underline{\lambda}_{T}\right\|^{2}}\right) \\
& \mathcal{F}_{42}^{\prime}=-\frac{\mu}{\left\|\underline{\lambda}_{T}\right\|}\left(\rho_{N} h \theta k^{w} r_{N} \lambda_{T_{1}} \frac{v_{T_{2}}}{\left\|\underline{v}_{T}\right\|}-\rho_{T} \lambda_{N} \frac{\lambda_{T_{1}} \lambda_{T_{2}}}{\left\|\underline{\lambda}_{T}\right\|^{2}}\right)
\end{aligned}
$$




$$
\begin{aligned}
& \mathcal{F}_{43}^{\prime}=-\mu \rho_{N} h \theta \frac{\lambda_{T_{1}}}{\left\|\underline{\lambda}_{T}\right\|} \\
& \mathcal{F}_{44}^{\prime}=\mu \frac{\lambda_{N}}{\left\|\underline{\lambda}_{T}\right\|} \frac{\lambda_{T_{2}}^{2}\left\|\underline{\lambda}_{T}\right\|^{2}}{\mathcal{F}_{45}^{\prime}}=-\mu \frac{\lambda_{N}}{\left\|\underline{\lambda}_{T}\right\|} \frac{\lambda_{T_{1}} \lambda_{T_{2}}}{\left\|\underline{\lambda}_{T}\right\|^{2}} \\
& \mathcal{F}_{46}^{\prime}=\mu \frac{\lambda_{T_{1}}}{\left\|\underline{\lambda}_{T}\right\|}\left(1-\rho_{N} h \theta k^{w}\left\|\underline{v}_{T}\right\|\right) \\
& \mathcal{F}_{51}^{\prime}=-\frac{\mu}{\left\|\underline{\lambda}_{T}\right\|}\left(\rho_{N} h \theta k^{w} r_{N} \lambda_{T_{2}} \frac{v_{T_{1}}}{\left\|\underline{v}_{T}\right\|}-\rho_{T} \lambda_{N} \frac{\lambda_{T_{1}} \lambda_{T_{2}}}{\left\|\underline{\lambda}_{T}\right\|^{2}}\right) \\
& \mathcal{F}_{52}^{\prime}=-\frac{\mu}{\left\|\underline{\lambda}_{T}\right\|}\left(\rho_{N} h \theta k^{w} r_{N} \lambda_{T_{2}} \frac{v_{T_{2}}}{\left\|\underline{v}_{T}\right\|}+\rho_{T} \lambda_{N} \frac{\lambda_{T_{1}}^{2}}{\left\|\underline{\lambda}_{T}\right\|^{2}}\right) \\
& \mathcal{F}_{53}^{\prime}=-\mu \rho_{N} h \theta \frac{\lambda_{T_{2}}}{\left\|\underline{\lambda}_{T}\right\|} \\
& \mathcal{F}_{54}^{\prime}=-\mu \frac{\lambda_{N}}{\left\|\underline{\lambda}_{T}\right\|} \frac{\lambda_{T_{1}} \lambda_{T_{2}}}{\left\|\underline{\lambda}_{T}\right\|^{2}} \\
& \mathcal{F}_{55}^{\prime}=\mu \frac{\lambda_{N}}{\left\|\underline{\lambda}_{T}\right\|} \frac{\lambda_{T_{1}}^{2}}{\left\|\underline{\lambda}_{T}\right\|^{2}} \\
& \mathcal{F}_{56}^{\prime}=\mu \frac{\lambda_{T_{2}}}{\left\|\underline{\lambda}_{T}\right\|}\left(1-\rho_{N} h \theta k^{w}\left\|\underline{v}_{T}\right\|\right) \\
& \mathcal{F}_{61}^{\prime}=-\rho_{N} h \theta k^{w} r_{N} \frac{v_{T_{1}}}{\left\|\underline{v}_{T}\right\|} \\
& \mathcal{F}_{62}^{\prime}=-\rho_{N} h \theta k^{w} r_{N} \frac{v_{T_{2}}}{\left\|\underline{v}_{T}\right\|}
\end{aligned}
$$

In this case, the two bodies have a sliding contact status.

\section{$\underline{\text { Comments, }}$}

* In theory, the real numbers $\rho_{N}$ and $\rho_{T}$ could take any positive values. But numerical reasons impose a restriction on their choice. To significantly reduce the number of iterations and allow for the convergence of the algorithm, we must minimize the conditioning of the tangent matrix $\mathcal{F}^{\prime}$. Inspired 
by the work of [13], we chose to take the following values

$$
\begin{gathered}
\rho_{N}=1 /\left(h W_{33}^{\alpha}\right) \\
\rho_{T}=2 /\left(h\left(W_{11}^{\alpha}+W_{22}^{\alpha}\right)\right)
\end{gathered}
$$

* When $\left\|\underline{v}_{T}\right\|$ is close to zero, the terms $\frac{v_{T_{i}}}{\left\|\underline{v}_{T}\right\|}$ for $i=1,2$ are replaced by zero.

* The wear displacements can not exceed the first layer of finite elements.

To go further, it would require mesh adaptation techniques. 
Compute mass matrix $M$

For each cycle do

Initialization $\dot{q}=0, \dot{q}^{e}=0, \dot{q}^{w}=0$

For each time step do

Compute gap $g_{N}^{-}$and local frames $\left(T_{1}, T_{2}, N\right)$

For each Newton iteration $(k)$ do

Compute tangent matrix $K_{T}$

Compute internal forces $F_{\text {int }}\left(q_{k}^{e+}\right)$

For each NSCD iteration (computation of $R_{k+1}$ ) do

For each node candidate to contact $(\alpha)$ do

Compute $v^{e \alpha}, r^{\alpha}$ (Local solver: 3.2)

Compute wear velocity $v_{N}^{w \alpha}=k^{w} r_{N}^{\alpha}\left|\underline{e}_{T}^{e \alpha}\right|$

Update $R_{k+1}$ with the new $r^{\alpha}$

Convergence test on reaction forces end For

end For

Compute $\dot{q}_{k+1}^{e+}$ (solution of eq. (14))

Compute $q_{k+1}^{e+}$

Convergence test

end For

Update velocity $\dot{q}^{+}=\dot{q}^{e+}+\dot{q}^{w+}$

Update positions $q^{+}, q^{e+}, q^{w+}$

end For

end For

Algorithm 1: Over view of the global algorithm 


\section{Numerical simulations : Total hip prosthesis}

The method developed in the previous paragraph, was programmed with MATLAB software. The example presented here was built to improve the approach. It concerns a total hip prosthesis wear. To deal with this type of problem some authors have adopted an experimental basis using hip simulators [7], [24], [2]. This type of approach allows quite accurate estimation of wear loss and location. However, it is hard to reproduce physiological loads and movements with these techniques. In this section, we propose to use our numerical method, while trying to be as close as possible to the actual relative motion of body contact and load conditions.

\subsection{Data set}

The femoral head prosthesis is modeled by a rigid body. For simplicity, only the spherical part of the head is considered. The acetabular cup is modeled by a half-spherical shell whose inner diameter is $28 \mathrm{~mm}$ and outer diameter is $56 \mathrm{~mm}$. The mesh contains 1562 tetrahedral elements (Figure 2). The cup is made of Ultra-High Molecular Weight Polyethylene (UHMWPE) with a Young modulus of $1016 \mathrm{MPa}$, a Poisson rate of 0.46 and a mass density of $938 \mathrm{Kg} / \mathrm{m}^{3}$. The friction coefficient is 0.07 .

According to Dowson [9], for a surface roughness of $0.01 \mu \mathrm{m}$ the wear factor in the presence of distilled water as an order of magnitude of $10^{-7}$ $\mathrm{mm}^{3} / \mathrm{Nm}$. Then, as in the study [11] the global wear factor $K$ is chosen to 
Figure 2: Total hip prosthesis model

be $2.210^{-7} \mathrm{~mm}^{3} / \mathrm{Nm}$. Assuming that the wear is only supported by the cup, one takes $K_{2}=K$ and $K_{1}$ is neglected. The local wear factor $k_{1}$ is computed according to element sizes (see finite element model (Figure 2)).

\subsection{Load conditions}

Based on the results of Paul [21], force $F$ due to weight and muscle actions, as a function of the flexion during gait, is modeled as a vertical force applied on one particular node of the upper face of the acetabular cup (see Figure 4). This is assessed by the following function

$$
\underline{F}(t)=\left(\frac{F_{\max }}{2}[1+\cos (4 \pi \mathrm{ft}+\pi)]+\mathrm{F}_{0}\right) \underline{\mathrm{z}}_{\mathrm{c}}
$$

The constant values $F_{\max }$ and $F_{0}$ are computed so as to obtain, for hight frequency $(f=100 \mathrm{~Hz})$, the resulting contact force, plotted in Figure 3, consistent with that reported in Saikko [26] (continuous line of Figure 7). We use an high frequency for load conditions in order to save computational time. The choice of the model (contact, friction, wear, large strains and dynamics), the boundary conditions (imposed forces), the mesh and the method of resolution, make that the time step allowing convergence must be about $10^{-5} \mathrm{~s}$. In addition, a cycle of walk is made in approximately one second, i.e. a frequency of loading of one Hertz. Then, a simulation of one cycle would require $10^{5}$ loading steps. Unfortunately, it is not possible with 
Figure 3: Load function (dashed line) and resulting contact reaction force for an amplification factor $n=1$ and a load frequency of $100 \mathrm{~Hz}$ (continuous line)

Figure 4: Load conditions during gait

our calculation. It is the reason why we use a load frequency of $100 \mathrm{~Hz}(1.000$ loading steps).

Displacements of nodes of the upper face of the cup are assumed to be nil in the directions $\underline{x}_{c}$ and $\underline{y}_{c}$. Thus, hip motion, through the motion of the upper face of the cup, is modeled by a slider of direction $\underline{z}_{c}$.

The center $O$ of the femoral head is blocked and the motion of the femur is simulated by applying rotations along the axis $\left(\underline{x}_{h}, \underline{y}_{h}, \underline{z}_{h}\right)$ (see Figure 4). The abduction-adduction angle is applied along the $\underline{x}_{h}$ axis, the flexionextension angle is applied along the $\underline{y}_{h}$ axis and the internal-external angle is applied along the $\underline{z}_{h}$ axis. The values of these rotation angles, as plotted in Figure 5, are deduced from the observations of Johnston and Smidt [14] .

\subsection{Results}

We aim to demonstrate that a single loading cycle, is enough to determine the volumetric wear rate and the worn area. For this, we based our numerical

Figure 5: Head rotations during gait 
Table 1: Volumetric wear comparisons and wear factor estimation after 1 million cycles for a given maximum load boundary force observations on experimental results.

The key point of the process is the choice of wear factor value. With a wear factor of $0.2210^{-6} \mathrm{~mm}^{3} / \mathrm{Nm}$ and for a single loading cycle, we find a wear volume loss of about $10.3410^{-06} \mathrm{~mm}^{3}$. Assuming the wear volume loss is linear versus the number of cycles (Archard's law), we obtain a wear rate of $10.34 \mathrm{~mm}^{3} / 10^{6}$ cycles. This numerical result is lower than that of our experimental reference of Saikko [26](see table 1). They found a wear rate between 11.3 and $17.6 \mathrm{~mm}^{3} / 10^{6}$ cycles.

Now, if we change the wear factor by taking $K_{1}=0.410^{-6} \mathrm{~mm}^{3} / \mathrm{Nm}$, which is an average of the values estimated by Saikko [26], we find $18.810^{-06}$ $\mathrm{mm}^{3}$ for a single loading cycle. This gives a wear rate of $18.8 \mathrm{~mm}^{3} / 10^{6}$ cycles. Our method thus allows us to estimate the wear rate. However, with this approach, it is impossible to determine the correct worn area and the correct wear depth after 1 million cycles. This is because the worn area and the wear depth are not linear versus the number of cycles. Indeed, Figure 9 represents the wear depth and the worn area after one cycle and differs markedly from the area that we should actually must find after 1 million cycles. A worn area and wear depth such as in Figure 8 should be found.

A strategy to accurately calculate the worn area and wear depth is to multiply the wear factor by an amplification factor $n$. In this case, with 
a number of cycles $c=\frac{10^{6}}{n}$ we can expect to obtain both the wear rate, the wear depth and the worn area. For example, if the wear coefficient is multiplied by an amplification factor $n=10$ or $10^{2} \ldots$ or $10^{6}$, the final result obtained after $c$ cycles $\left(c=10^{5}\right.$ or $10^{4} \ldots$ or 1$)$ represents the result obtained after $10^{6}$ cycles with an amplification factor $n=1$.

Applying this strategy, an estimate of the wear rate depending on the amplification factor is plotted in the top of Figure 6. This series of calculations was carried out with a boundary load function (not plotted in this paper) adapted for an amplification factor $n=1$. We find that the wear rate increases with the amplification factor. This increase of wear rate comes from an increase of the resulting contact force (bottom of Figure 6). The dashed line of Figure 7 gives an illustration, during one cycle, of this increase of contact forces. This curve is the result of a simulation done with an amplification factor $n=10^{6}$ and with the boundary load function adapted to $n=1$. This overload in the resulting contact forces is explained by inertia effects due to the hight load frequency we used $(100 \mathrm{~Hz})$. Indeed, as the wear coefficient is increased, the wear displacements are more important and thus accelerations too.

To overcome this difficulty, we modify the boundary load intensity. For an amplification factor $n=10^{6}$, we use the boundary load function plotted with dashed line in Figure 3 so as to have a resulting contact force consistent with that reported in Saikko [26] (continuous line of Figure 7). With these 
Figure 6: Wear rate (top) and contact force (bottom) versus the amplification factor

Figure 7: Resulting contact force for an amplification factor $n=10^{6}$ and a load frequency of $100 \mathrm{~Hz}$ (dashed line) compared with the reference contact force (Saikko [26]) (continuous line)

boundary conditions, the computed wear rate is about $18.88 \mathrm{~mm}^{3} / 10^{6}$ cycles and the worn area is consistent with experimental observations (see Figure $8)$.

\section{Discussion}

Several comments could be made on the results we have presented, with the following being the most important.

* The wear rate we computed was greater than that measured experimentally by Saikko [26]. This is due to the kinematics that we imposed on the femoral head. We chose a head movement close the physiological kinematics, while Saikko's tests [26] were conducted on a hip simulator, which is different. In the paper [25], tracks made by contact points were compared between

Figure 8: Wear depth $(\mathrm{mm})$ obtained after one cycle with an amplification factor $n=10^{6}$, the loading force plotted in dashed line of Figure 3 and a load frequency of $100 \mathrm{~Hz}$ 
Figure 9: Wear depth $(\mathrm{mm})$ obtained after one cycle with an amplification factor $n=1$, the loading force plotted in dashed line of Figure 3 and a load frequency of $100 \mathrm{~Hz}$

different simulators and physiological tracks. It was noted that the distance rubbed was greater in the case of a physiological drive, which explains why wear volume is higher in our calculations.

* It should also be noted that to achieve satisfactory results, we used a wear factor approximately twofold higher than that proposed by [11]. We made this choice for two reasons. The first is mentioned in the preceding paragraph, i.e. it is an average of the values found by Saikko [26] and its work is our experimental reference study. The second reason, which is physical evidence, is that many studies have shown that a change of direction of friction is an aggravating factor for wear (see [19], [29]). But in the work of [11], there is no change of direction, unlike the friction trajectories in the hip joint. Due to these changes of directions, it is necessary to use a wear factor higher than that measured in [11].

* In Figure 7 we observe the effects of our fully coupled method. Indeed, one can note that the peaks of resulting contact forces are different during one cycle. This difference is significant with an amplification factor $n=10^{6}$. The second peak is higher than the first one. It less perceptible and is even slightly reversed with $n=1$ (Figure 3 ). It is an illustration of the nonlinearity due to the impact of the wear on the contact area and stresses. 
Finally, we highlighted that, for a high amplification factor, the inertia effects increase the estimated wear rate. These inertia effects are due to the high solicitation frequency that we have chosen. This choice, which does not correspond to reality, allows us to simulate a load cycle in about $2 \mathrm{~h}$ on a PC with a time step of $2.510^{-5} \mathrm{~s}$. If we were to simulate the real phenomenon with a load frequency of $1 \mathrm{~Hz}$, it would require a 100-fold greater calculation time. This is why we chose a high frequency and limited the inertia effects by changing the loading conditions while providing an intensity of the resulting contact force in accordance with experience. It should be noted that the time step is limited not only because of the wear method but also because of the driving force conditions from the cup. Due to the solid rigid movements of the cup, the calculation requires small time steps. The same problem, under driven displacement conditions, allows a 10-fold greater time step.

\section{Conclusion}

Through the results presented in the previous paragraph, our method is clearly able to quite accurately simulate the wear phenomenon in this hip prosthesis example. However, there are other approaches that provide numerical results. These include the works of [11], [20], [22], [18], [27], but they require many loading cycles and the geometry has to be updated as little as possible in order to avoid delaying the calculations. Conversely, our method can simulate the process in a single load cycle because the algorithm takes 
the geometric variations at each iteration into account.

Obviously, our method has its limitations because of simplifying assumptions that we made. As the model is currently constructed, the wear displacements can not exceed the first layer of finite elements. To go further, it would require mesh adaptation techniques. More over, wear cannot be too great because we neglect the mass loss in the inertia effects terms. Our method is illustrated with a simplest wear model and a pure elastic behaviour, but could be straightforwardly extended to non-linear bulk behaviour and to more complex wear laws. Actually, the model is based on Archard's law which imposes a linearity wear rate versus the number of cycles. But many works, such as [23] , [12], show that this relationship is not linear. The model could be enhanced by taking variations in the wear factor into account to estimate wear beyond 1 million cycles.

Finally, in order to provide hospital practitioners with real answers on hip prostheses it would be necessary to more accurately model the load distribution due to the action of muscles and ligaments.

\section{References}

[1] Adélaïde L., Jourdan F., Bohatier C., (2003), Frictional contact solver and mesh adaptation in spaceñtime finite element method, European Journal of Mechanics - A/Solids, Vol. 22, Issue 4, Pages 633-647 
[2] Affatato S., Bordini B., Fagnano C., Taddei P., Tinti A., Toni A. (2002), Effects of the sterilisation method on the wear of UHMWPE acetabular cups tested in a hip joint simulator, Biomaterials, Vol. 23, pp. 14391446

[3] Agelet de Saracibar C., Chiumenti M. (1999), On the numerical modeling of frictional wear phenomena, Comp. Meth. Appl. Mech. Engrg. Vol. 177, pp. 401-426

[4] Alart P. and Curnier A. (1991), A mixed formulation for frictional contact problems prone to Newton like solution method, Comput. Methods Appl. Mech. Engrg. , Vol. 92, n3, pp. 353-375

[5] Archard J. F. (1953) Contact and rubbing of flat surfaces, J. Appl. Phys. 24 (8), pp. 981-988

[6] Bingley M.S. and Schnee S. (2005), A study of the mechanisms of abrasive wear for ductile metals under wet and dry three-body conditions, Wear, Vol. 258, pp. 50-61

[7] Bowsher J. G., Shelton J.C. (2001), A hip simulator study of the influence of patient activity level on the previous wear of crosslinked polyethylene under smooth and roughened femoral conditions, Wear, Vol. 250, pp. $167 \tilde{n} 179$ 
[8] Cho H.J., Wei W.J., Kao H.C. and Cheng C.K. (2004), Wear behavior of UHMWPE sliding on artificial hip arthroplasty materials, Materials Chem. And Phys., Vol. 88, pp. 9-11

[9] Dowson D. (1995), A comparative study of the performance of metallic and ceramic femoral head components in total replacement hip joints, Wear, Vol. 190, pp. 171-183

[10] Dragon-Louiset M., Stolz C. (1999) Approche thermodynamique des phénomènes liés à l'usure de contact, C. R. Acad. Sci. Paris, t. 327, Serie II b, PP. 1275-1280

[11] Fregly B.J, Sawyer W. G., Harman M. H., Scott A. Banks S. A. (2005) Computational wear prediction of a total knee replacement from in vivo kinematics, Jour. of Biomechanics, Vol. 38, pp. 305-314

[12] Galvin A. L., et al., (2005), Comparison of wear of ultra high molecular weight polyethylene acetabular cups against alumina ceramic and chromium nitride coated femoral heads, Wear, Volume 259, Issues 7-12, pp. $972-976$

[13] Jean M. (1999), The non-smooth contact dynamics method, Comp. Meth. Appl. Mech. Engrg. Vol. 177, pp. 235-257

[14] Johnston R.C. and Smidt G.L. (1969), Measurement of hipñjoint motion during walkingóevaluation of an electrogoniometric method. The 
Journal of Bone and Joint Surgery, Vol. 51ñA, pp. 1083ñ1094.

[15] Jourdan F. (2006), Numerical wear modeling in dynamics and large strains : Application to knee joint prostheses, Wear, Vol. 261, pp 283292

[16] Jourdan F., Alart P., Jean M. (1998) A Gauss Seidel like algorithm to solve frictional contact problems, Comput. Methods Appl. Mech. Engrg., Vol. 155, pp. 31-47

[17] Jourdan F., Alart P., Jean M., (1998) An alternative method between implicit and explicit schemes devoted to frictional contact problems in deep drawing simulation Journal of Materials Processing Technology, Volumes 80-81, Pages 257-262

[18] Knight L. A., Pal S., Coleman J. C., Bronson F., Haider H., Levine D. L., Taylor M., Rullkoetter P. J., (2007), Comparison of long-term numerical and experimental total knee replacement wear during simulated gait loading, Journal of Biomechanics, Vol. 40, Issue 7, pp. 1550-1558

[19] Laurent M. P., Johnson T. S., Yao J. Q., Blanchard C. R., Crowninshield R. D., (2003), In vitro lateral versus medial wear of a knee prosthesis, Wear, Vol 255, pp. 1101-1106

[20] Öqvist M. (2001), Numerical simulations of mild wear using updated geometry with different step size approaches, Wear, Vol. 249, pp. 6-11 
[21] Paul J.P. (1976), Force actions transmitted by joints in the human body, Proceedings of the Royal Society of London B, Vol. 192, pp. $163-172$

[22] Põdra P., Andersson S., (1997), Wear simulation with the Winkler surface model, Wear, Vol. 207, pp. 79-85

[23] Rieker C. B., Sch^n R., Konrad R., Liebentritt G., Gnepf P., Shen , Roberts P., Grigoris P., (2005), Influence of the Clearance on In-Vitro Tribology of Large Diameter Metal-on-Metal Articulations Pertaining to Resurfacing Hip Implants, Orthopedic Clinics of North America, Volume 36, Issue 2, pp. 135-142

[24] Saikko V., Ahlroos T., Calonius O, Ker\%onen J., (2001), Wear simulation of total hip prostheses with polyethylene against $\mathrm{CoCr}$, alumina and diamond-like carbon, Biomaterials, Vol. 22, pp. 1507ñ1514

[25] Saikko V., Calonius O., (2002), Slide track analysis of the relative motion between femoral head and acetabular cup in walking and in hip simulators, Journal of Biomechanics, Volume 35, Issue 4, pp. 455-464

[26] Saikko V., Calonius O., (2003), An improved method of computing the wear factor for total hip prostheses involving the variation of relative motion and contact pressure with location on the bearing surface, Journal of Biomechanics, Vol. 36, pp. 1819ñ1827 
[27] Sfantos G.K., Aliabadi M.H., (2007), A boundary element formulation for three-dimensional sliding wear simulation, Wear, Volume 262, Issues 5-6, pp. $672-683$

[28] Stromberg N., (1998) Finite element treatment of two-dimensional thermoelastic wear problems, Comp. Meth. Appl. Mech. Engrg. Vol. 177, pp. $441-455$

[29] Turell M., Wang A., Bellare A., (2003), Quantification of the effect of cross-path motion on the wear rate of ultra-high molecular weight polyethylene, Wear, Volume 255, Issues 7-12, pp. 1034-1039

[30] Wang A. (2001), A unified theory of wear for ultra-high molecular weight polyethylene in multi-directional sliding, Wear, Vol. 248, pp. $38-47$

[31] Zmitrowics A. (1987) A thermodynamical model of contact, friction and wear : Governing equations, Wear 114, pp. 135-168 